\title{
PRODUCT INFORMATION
}

\section{TYSABRI $^{\circledR}$ (natalizumab, rmc)}

\begin{abstract}
WARNING
TYSABRI ${ }^{\circledR}$ is associated with an increased risk of progressive multifocal leucoencephalopathy (PML), an opportunistic viral infection of the brain that usually leads to death or severe disability. Healthcare professionals should monitor patients on TYSABRI for any new signs or symptoms that may be suggestive of PML. TYSABRI dosing should be withheld immediately at the first signs or symptoms suggestive of PML. For diagnosis, an evaluation that includes a gadolinium-enhanced magnetic resonance imaging (MRI) scan of the brain and when indicated, cerebrospinal fluid analysis for JC viral DNA are recommended (see CONTRAINDICATIONS and PRECAUTIONS, Progressive Multifocal Leucoencephalopathy).
\end{abstract}

\section{NAME OF THE MEDICINE}

TYSABRI (natalizumab) is a recombinant humanised IgG4 monoclonal antibody produced in murine myeloma cells. Natalizumab $(\mathrm{rmc})$ contains human framework regions and the complementarity-determining regions of a murine antibody that binds to $\alpha 4$-integrin. The molecular weight of natalizumab is 149 kilodaltons.

\section{DESCRIPTION}

TYSABRI (natalizumab) is supplied as a sterile, colourless, clear to slightly opalescent concentrated solution for intravenous (IV) infusion. Each $15 \mathrm{~mL}$ dose contains $300 \mathrm{mg}$ natalizumab. TYSABRI also contains $123 \mathrm{mg}$ sodium chloride, $17 \mathrm{mg}$ sodium phosphate monobasic monohydrate, $7.24 \mathrm{mg}$ sodium phosphate dibasic heptahydrate and $3 \mathrm{mg}$ polysorbate 80 in water for injections, at pH 6.1.

\section{PHARMACOLOGY}

\section{Pharmacodynamics}

TYSABRI binds to the $\alpha 4$-subunit of $\alpha 4 \beta 1$ and $\alpha 4 \beta 7$ integrins expressed on the surface of all leucocytes except neutrophils, and inhibits the $\alpha 4$-mediated adhesion of leucocytes to their counter receptor(s). The receptors for the $\alpha 4$ family of integrins include vascular cell adhesion molecule-1 (VCAM-1), which is expressed on activated vascular endothelium, and mucosal addressin cell adhesion molecule-1 (MadCAM-1) present on vascular endothelial cells of the gastrointestinal tract. Disruption of these molecular interactions prevents transmigration of leucocytes across the endothelium into inflamed parenchymal tissue. In vitro, anti- $\alpha 4$-integrin antibodies also block $\alpha 4$-mediated cell binding to ligands such as osteopontin and an alternatively spliced domain of fibronectin, connecting segment-1 (CS-1).

The specific mechanism(s) by which TYSABRI exerts its effects in multiple sclerosis have not been fully defined. In multiple sclerosis, lesions are believed to occur when activated inflammatory cells, including T-lymphocytes, cross the blood-brain barrier (BBB). Leucocyte migration across the BBB involves interaction between adhesion molecules on inflammatory cells, and their counter-receptors present on endothelial cells of the vessel wall. The clinical effect of natalizumab in multiple sclerosis may be secondary to blockade of the molecular 
interaction of $\alpha 4 \beta 1$-integrin expressed by inflammatory cells with VCAM-1 on vascular endothelial cells, and with CS-1 and/or osteopontin expressed by parenchymal cells in the brain. Data from an experimental autoimmune encephalitis animal model of multiple sclerosis demonstrate reduction of leucocyte migration into brain parenchyma and reduction of plaque formation detected by magnetic resonance imaging (MRI) following repeated administration of natalizumab. The clinical relevance of these animal data is unknown.

TYSABRI administration increases the number of circulating leucocytes, (including lymphocytes, monocytes, basophils, and eosinophils) due to inhibition of transmigration out of the vascular space. TYSABRI does not affect the number of circulating neutrophils (see PRECAUTIONS, Effect on Laboratory Tests).

\section{Pharmacokinetics}

Following the repeat intravenous administration of a $300 \mathrm{mg}$ dose of natalizumab to patients with multiple sclerosis (MS), the mean ( \pm standard deviation) maximum observed serum concentration was $110 \pm 52 \mu \mathrm{g} / \mathrm{mL}$. Mean average steady-state trough concentrations ranged from $23 \mu \mathrm{g} / \mathrm{mL}$ to $29 \mu \mathrm{g} / \mathrm{mL}$. The observed time to steady-state was approximately 24 weeks after every 4 weeks of dosing. The mean ( \pm standard deviation) half-life, volume of distribution and clearance of natalizumab were $11 \pm 4$ days, $5.7 \pm 1.9 \mathrm{~L}$ and $16 \pm 5 \mathrm{~mL} / \mathrm{hour}$, respectively.

The effects of covariates such as body weight, age, gender, and presence of antinatalizumab antibodies on natalizumab pharmacokinetics were investigated in a population pharmacokinetic study. Natalizumab clearance increased with body weight in a less than proportional manner, such that a $43 \%$ increase in body weight resulted in a $32 \%$ increase in clearance. The presence of persistent anti-natalizumab antibodies increased natalizumab clearance approximately 3-fold (see ADVERSE EFFECTS, Immunogenicity). Age (18 to 62 years) and gender did not influence natalizumab pharmacokinetics.

Study 101MS101 was an open label, randomised, crossover study in 43 subjects with relapsing forms of multiple sclerosis comparing the pharmacokinetic properties of natalizumab produced by the initial manufacturing process and natalizumab manufactured using the high titre process. Nearly identical concentration/time profiles were observed. Comparability of both manufacturing methods was well demonstrated.

The effect of plasma exchange on natalizumab clearance and pharmacodynamics was evaluated in a study of 12 MS patients. Estimates of the total drug removal after 3 plasma exchanges (over a 5-8 day interval) was approximately 70-80\%. This compares to approximately $40 \%$ seen in earlier studies in which measurements occurred after drug discontinuation over a similar period of observation. The impact of plasma exchange on the restitution of lymphocyte migration and ultimately its clinical usefulness is unknown.

The pharmacokinetics of TYSABRI in paediatric or adolescent MS patients younger than 18 years, or patients with renal or hepatic insufficiency have not been studied.

\section{CLINICAL TRIALS}

TYSABRI was evaluated in two randomised, double-blind, placebo-controlled trials in patients with relapsing remitting multiple sclerosis. Both studies enrolled patients who experienced at least one clinical relapse during the prior year and had a Kurtzke Expanded Disability Status Scale (EDSS) score between 0 and 5.0. Patients with primary progressive, secondary progressive and progressive relapsing MS were excluded from these trials.

In both studies, neurological evaluations were performed every 12 weeks and at times of suspected relapse. Magnetic resonance imaging evaluations for T1-weighted gadolinium 
(Gd)-enhancing lesions and T2-hyperintense lesions were performed annually.

Study 1 enrolled patients who had not received any interferon-beta or glatiramer acetate for at least the previous 6 months; approximately 94\% had never been treated with these agents. Median age was 37, with a median disease duration of 5 years. Patients were randomised in a 2:1 ratio to receive TYSABRI $300 \mathrm{mg}$ IV infusion $(n=627)$ or placebo $(n=315)$ every 4 weeks for up to 28 months (30 infusions).

Study 2 enrolled patients who had experienced one or more relapses while on treatment with AVONEX $^{\circledR}$ (interferon beta-1a) $30 \mu \mathrm{g}$ intramuscularly (IM) once weekly during the year prior to study entry. Median age was 39, with a median disease duration of 7 years. Patients were evenly randomised to receive TYSABRI $300 \mathrm{mg}(n=589)$ or placebo $(n=582)$ every 4 weeks for up to 28 months (30 infusions). All patients continued to receive AVONEX $30 \mu \mathrm{g}$ IM once weekly. In this study, TYSABRI in combination with AVONEX was compared with AVONEX alone.

In both studies, there were two pre-specified primary endpoints, annualised clinical relapse rate at one year and disease progression, measured by Extended Disability Severity Scale (EDSS), at two years. Sustained increase in disability was defined as an increase of at least 1 point on the EDSS from baseline EDSS $\geq 1.0$ that was sustained for 12 weeks, or at least a 1.5 point increase on the EDSS from baseline EDSS $=0$ that was sustained for 12 weeks. Results are shown in Tables 1 and 2. Median time on study drug was 120 weeks in each study.

Time to onset of sustained increase in disability was longer in patients treated with TYSABRI than in patients treated with placebo in Studies 1 (Figure 1) and 2. The proportions of patients with increased disability and annualised relapse rate were also lower in patients treated with TYSABRI than in patients treated with placebo in Studies 1 and 2. Subgroup and sensitivity analyses showed results consistent with the primary analyses. The sensitivity analysis of increase in disability that was sustained for 24 weeks yielded a $54 \%$ reduction in the TYSABRI group in Study $1(p<0.001)$.

Changes in MRI findings often do not correlate with changes in the clinical status of patients (e.g. disability progression). The prognostic significance of the MRI findings in these studies has not been evaluated.

Table 1. Clinical and MRI Endpoints in Study 1 (Monotherapy Study) at 2 Years

\begin{tabular}{|c|c|c|}
\hline & $\begin{array}{c}\text { TYSABRI } \\
n=627\end{array}$ & $\begin{array}{l}\text { Placebo } \\
n=315\end{array}$ \\
\hline \multicolumn{3}{|l|}{ Clinical Endpoints } \\
\hline Percentage with sustained increase in disability & $17 \%$ & $29 \%$ \\
\hline Relative Risk Reduction & \multicolumn{2}{|c|}{$42 \%(95 \% \mathrm{Cl} 23 \%, 57 \%)$} \\
\hline Annualised relapse rate & 0.23 & 0.73 \\
\hline Relative reduction (percentage) & \multicolumn{2}{|c|}{$68 \%$ (95\% Cl 60\%, 74\%) } \\
\hline Percentage of patients remaining relapse-free & $67 \%$ & $41 \%$ \\
\hline \multicolumn{3}{|l|}{ MRI Endpoints } \\
\hline $\begin{array}{l}\text { New or newly enlarging T2-hyperintense lesions } \\
\text { Median } \\
\text { Percentage of patients with:* }\end{array}$ & 0 & 5 \\
\hline 0 lesions & $57 \%$ & $15 \%$ \\
\hline 1 lesion & $17 \%$ & $10 \%$ \\
\hline 2 lesions & $8 \%$ & $8 \%$ \\
\hline 3 or more lesions & $18 \%$ & $68 \%$ \\
\hline
\end{tabular}




\begin{tabular}{l|cc} 
& $\begin{array}{c}\text { TYSABRI } \\
\mathrm{n}=627\end{array}$ & $\begin{array}{c}\text { Placebo } \\
\mathrm{n}=315\end{array}$ \\
\hline $\begin{array}{l}\text { Gd-enhancing lesions } \\
\text { Median }\end{array}$ & 0 & 0 \\
Percentage of patients with: & $97 \%$ & $72 \%$ \\
$\quad$ lesions & $2 \%$ & $12 \%$ \\
1 lesion & $1 \%$ & $16 \%$ \\
\hline or more lesions & & \\
\hline
\end{tabular}

All analyses were intent-to-treat. For each endpoint, $p<0.001$. Determination of $p$-values: Increase in disability by Cox proportional hazards model adjusted for baseline EDSS and age; relapse rate by Poisson regression adjusting for baseline relapse rate, EDSS, presence of Gd-enhancing lesions, age; percentage relapse-free by logistic regression adjusting for baseline relapse rate; and lesion number by ordinal logistic regression adjusting for baseline lesion number.

* Values do not total $100 \%$ due to rounding.

Table 2. Clinical and MRI Endpoints in Study 2 (Add-On Study) at 2 Years

\begin{tabular}{|c|c|c|}
\hline & $\begin{array}{c}\text { TYSABRI } \\
\text { plus AVONEX } \\
n=589\end{array}$ & $\begin{array}{c}\text { Placebo } \\
\text { plus AVONEX } \\
n=582\end{array}$ \\
\hline \multicolumn{3}{|l|}{ Clinical Endpoints } \\
\hline Percentage with sustained increase in disability & $23 \%$ & $29 \%$ \\
\hline Relative Risk Reduction & \multicolumn{2}{|c|}{$24 \%(95 \% \mathrm{Cl} 4 \%, 39 \%)$} \\
\hline Annualised relapse rate & 0.34 & 0.75 \\
\hline Relative reduction (percentage) & \multicolumn{2}{|c|}{$55 \%(95 \% \mathrm{Cl} 47 \%, 62 \%)$} \\
\hline Percentage of patients remaining relapse-free & $54 \%$ & $32 \%$ \\
\hline \multicolumn{3}{|l|}{ MRI Endpoints } \\
\hline \multicolumn{3}{|l|}{ New or newly enlarging T2-hyperintense lesions } \\
\hline Median & 0 & 3 \\
\hline \multicolumn{3}{|l|}{ Percentage of patients with:* } \\
\hline 0 lesions & $67 \%$ & $30 \%$ \\
\hline 1 lesion & $13 \%$ & $9 \%$ \\
\hline 2 lesions & $7 \%$ & $10 \%$ \\
\hline 3 or more lesions & $14 \%$ & $50 \%$ \\
\hline \multicolumn{3}{|l|}{ Gd-enhancing lesions } \\
\hline Median & 0 & 0 \\
\hline \multicolumn{3}{|l|}{ Percentage of patients with:* } \\
\hline 0 lesions & $96 \%$ & $75 \%$ \\
\hline 1 lesion & $2 \%$ & $12 \%$ \\
\hline 2 or more lesions & $1 \%$ & $14 \%$ \\
\hline
\end{tabular}

All analyses were intent-to-treat. For disability progression $p=0.024$, for all other endpoints, $p<0.001$. Determination of $p$-values: increase in disability by Cox proportional hazards model adjusted for baseline EDSS; relapse rate by Poisson regression adjusting for baseline relapse rate, EDSS, presence of Gd-enhancing lesions, age; percentage relapse-free by logistic regression adjusting for baseline relapse rate; and lesion number by ordinal logistic regression adjusting for baseline lesion number.

*Values do not total $100 \%$ due to rounding. 
Figure 1. Time to Increase in Disability Sustained for 12 Weeks in Study 1

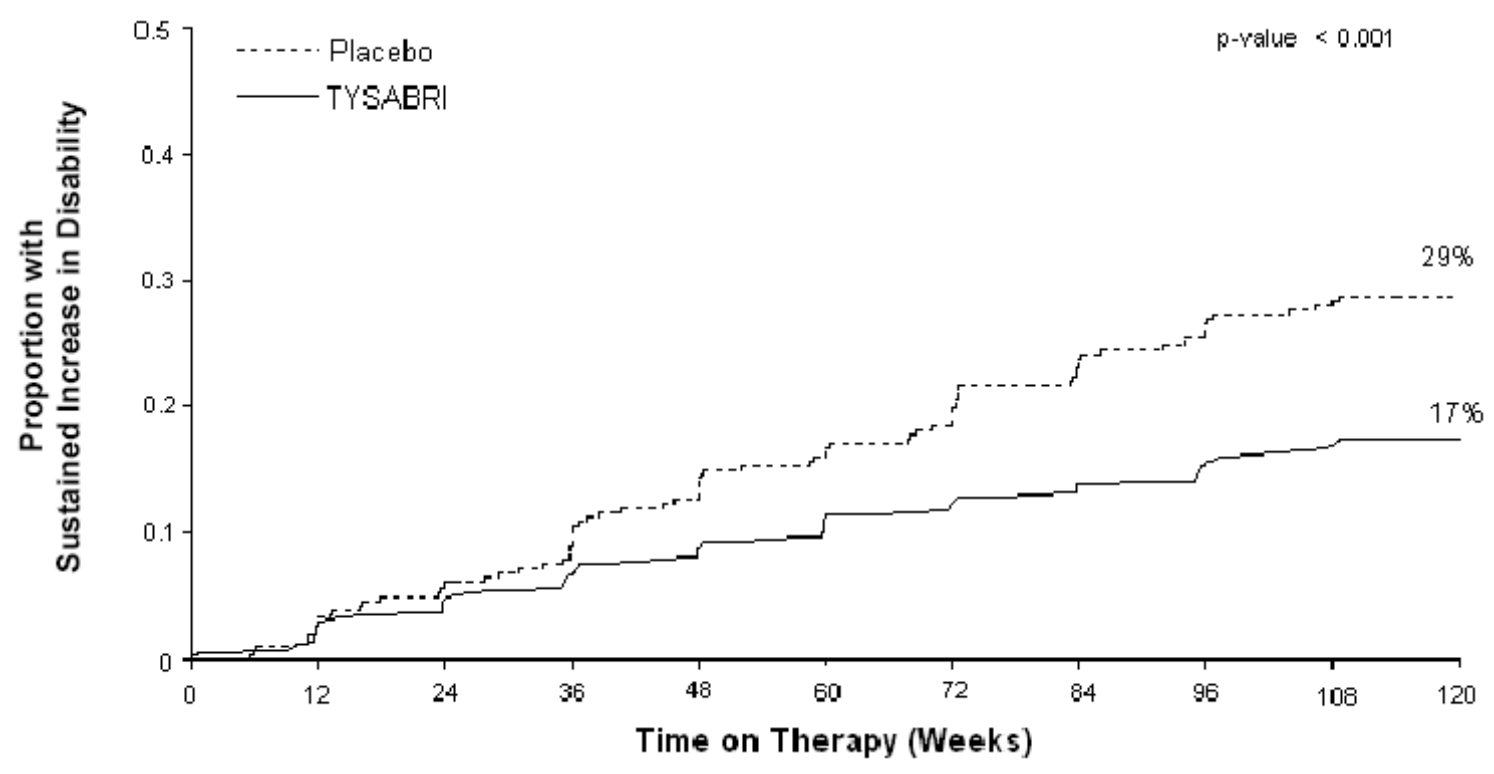

Study 101MS101 was an open label, randomised, crossover study in 43 patients with relapsing forms of multiple sclerosis comparing the pharmacokinetic properties of natalizumab produced by the initial manufacturing process and natalizumab manufactured using the high titre process. As a secondary endpoint the tolerability and safety of both manufacturing processes were assessed. No notable differences were observed in the overall incidence for any safety measures in patients receiving either natalizumab preparation. The safety profile over this 36 week study was similar to that described in other natalizumab studies.

Study 101MS201 was a mulitcentre, open-label, repeat-dose study of 113 natalizumab naïve patients with relapsing forms of multiple sclerosis assessing the immunogenicity of $300 \mathrm{mg}$ of natalizumab manufactured using the high titre process. Natalizumab was administered intravenously over 60 minutes every 4 weeks for 9 months (a total of 9 doses). A secondary objective was to evaluate safety. All subjects received at least one dose of natalizumab. Natalizumab manufactured using the high titre process was well tolerated with an adverse event profile similar to that observed in previous clinical studies. Persistent anti-natalizumab antibodies formed in a small number of subjects which is comparable to the rate observed with previous clinical studies.

\section{INDICATIONS}

TYSABRI is indicated as monotherapy for the treatment of patients with relapsing remitting multiple sclerosis (MS) to delay the progression of physical disability and to reduce the frequency of relapse.

\section{CONTRAINDICATIONS}

TYSABRI should not be administered to patients with known hypersensitivity to natalizumab or any of the excipients, or to patients with known hypersensitivity to murine derived proteins.

TYSABRI is contraindicated in patients who have or have had progressive multifocal leucoencephalopathy (PML).

TYSABRI should not be administered to patients with increased risk for opportunistic infections, including those immunocompromised due to current or recent immunosuppressive therapies 
(e.g. azathioprine, mitozantrone), or systemic medical conditions resulting in significantly compromised immune system function (e.g. human immunodeficiency virus, organ transplant, active malignancy).

TYSABRI should not be administered in combination with immunomodulatory agents (e.g. beta interferons or glatiramer acetate).

\section{PRECAUTIONS}

TYSABRI therapy is to be initiated and supervised by neurologists, in centres with timely access to MRI. Prescribing neurologists must discuss the benefits and risks of TYSABRI therapy with the patient, provide them with the Consumer Medicine Information and a Patient Alert Card and obtain individual, written, fully informed consent from the patient (or legal guardian where appropriate) for the use of TYSABRI. Neurologists should counsel patients on the importance of uninterrupted dosing, particularly in the early months of treatment (see Hypersensitivity).

The Alert Card reminds patients that because of the risks of PML and opportunistic infections with TYSABRI, they must contact their doctor if they have unusual or prolonged new neurological symptoms or if they have severe or prolonged symptoms of infection. Patients should be instructed that they should inform all their healthcare providers that they are receiving treatment with TYSABRI.

The neurologist should reevaluate the patient 3 months after the first infusion, 6 months after the first infusion and every 6 months thereafter. Continued therapy must be carefully reconsidered in patients who show no evidence of therapeutic benefit beyond 6 months. Data on the safety and efficacy of TYSABRI at two years were generated from controlled, double-blind studies. Post-marketing data are available for use up to six years, however continued therapy beyond two years should be considered only following a reassessment of the potential for benefit and risk in patients who are known to be anti-JCV antibody positive.

\section{Progressive Multifocal Leucoencephalopathy (PML)}

Use of TYSABRI has been associated with an increased risk of PML (see BOXED WARNING and ADVERSE EFFECTS). The absolute risk for PML in patients treated with TYSABRI cannot be precisely estimated. There are no known interventions that can reliably prevent PML or adequately treat PML if it occurs.

The following risk factors are associated with an increased risk of PML.

- The presence of anti-JCV antibodies.

- Treatment duration, especially beyond 2 years in patients who are anti-JCV antibody positive. There is limited experience in patients who have received more than 6 years of TYSABRI treatment, therefore, the risk of PML in these patients cannot currently be estimated.

- Immunosuppressant use prior to receiving TYSABRI.

Anti-JCV antibody status identifies different levels of risk for PML in TYSABRI treated patients. Patients who are anti-JCV antibody positive are at increased risk of developing PML compared to patients who are anti-JCV antibody negative. Patients who are anti-JCV antibody negative are at a significantly lower risk. Anti-JCV antibody negative patients may still be at risk of PML for reasons such as a new JCV infection, fluctuating antibody status or a false negative test result.

Therefore, testing should be carried out prior to initiating TYSABRI therapy or in patients already receiving TYSABRI in whom antibody status is unknown. Testing should be performed using an anti-JCV antibody assay that has been analytically validated in MS 
patients. Six-monthly testing of patients who are anti-JCV antibody negative is also recommended.

Anti-JCV antibody assays should not be used to diagnose PML. Anti-JCV antibody testing should not be performed during or for at least two weeks following plasma exchange due to the removal of antibodies from the serum.

Patients who have all three risk factors for PML (i.e., are anti-JCV antibody positive and have received more than 2 years of TYSABRI therapy and have received prior immunosuppressant therapy ) have a significantly higher risk of PML as determined during studies using a two-step ELISA anti-JCV antibody assay, although the relative risk may vary using other assays. The risks and benefits of continuing treatment with TYSABRI should be carefully considered in patients who have all three of these risk factors for PML. Data beyond 6 years of treatment are limited (see Post-Marketing Experience). Before initiation of treatment with TYSABRI, a recent (usually within 3 months) Magnetic Resonance Image (MRI) should be available as a reference, and be repeated on a yearly routine basis to update this reference. This MRI may be helpful in differentiating subsequent multiple sclerosis symptoms from PML.

Patients must be monitored at regular intervals for any new or worsening neurological symptoms or signs that may be suggestive of PML. If new neurological symptoms suggestive of PML occur, further dosing is to be suspended until PML has been excluded. If any doubt exists, further evaluation, including MRI scan (compared with pre-treatment and annual MRI), CSF testing for JC Viral DNA and repeat neurological assessments, should be considered. If initial investigations prove negative, but clinical suspicion for PML still remains, TYSABRI should not be restarted and repeat investigations should be undertaken. Once the clinician has excluded PML, dosing of TYSABRI may resume.

The physician should be particularly alert to symptoms suggestive of PML that the patient may not notice (e.g. cognitive or psychiatric symptoms). Patients should also be advised to inform their partner or caregivers about their treatment, since they may notice symptoms that the patient is not aware of.

If a patient develops PML, the dosing of TYSABRI must be permanently discontinued to enable reconstitution of the immune system.

\section{PML and IRIS (Immune Reconstitution Inflammatory Syndrome)}

In TYSABRI patients that develop PML, IRIS occurs in almost all cases after withdrawal or removal of TYSABRI, e.g. by plasma exchange (see Pharmacokinetics). IRIS is thought to result from the restoration of immune function in patients with PML. IRIS presents as a worsening in neurological status that may be rapid, which can lead to serious neurological complications and may be fatal. Monitoring for development of IRIS, which has occurred within days to several weeks after plasma exchange in TYSABRI treated patients with PML, and appropriate treatment of the associated inflammation during recovery from PML should be undertaken.

\section{Other Opportunistic Infections}

Other opportunistic infections have been reported with the use of TYSABRI, primarily in patients with Crohn's disease who were immunocompromised or where significant co-morbidity existed (see ADVERSE EFFECTS). However increased risk of other opportunistic infections with use of TYSABRI in patients without these co-morbidities cannot currently be excluded.

Physicians should be aware of the possibility that other opportunistic infections may occur during TYSABRI therapy and should include them in the differential diagnosis of infections that occur in TYSABRI-treated patients. If an opportunistic infection is suspected, dosing 
with TYSABRI is to be suspended until such infections can be excluded through further evaluations.

If a patient receiving TYSABRI develops an opportunistic infection, dosing of TYSABRI must be permanently discontinued.

\section{Hepatotoxicity}

Spontaneous suspect adverse drug reactions of liver injury, including severe liver injury, have been reported from the market. Signs of liver injury, including markedly elevated serum hepatic enzymes and elevated total bilirubin, occurred as early as six days after the first dose. Signs of liver injury have also been reported for the first time after multiple doses, including cases with rechallenge. In these patients recovery of liver function occurred following cessation of therapy.

If liver injury occurs during treatment with TYSABRI the drug should be discontinued and investigation of cause undertaken. TYSABRI should be initiated with caution in patients with a history of liver disease and liver function tests should be regularly monitored in these patients.

\section{Stopping TYSABRI Therapy - Prolonged Pharmacodynamic Effects}

If a decision is made to stop treatment with TYSABRI, the physician needs to be aware that natalizumab remains in the blood, and may have pharmacodynamic effects (e.g. increased lymphocyte counts) for approximately 12 weeks following the last dose. Starting other therapies during this interval may result in a concomitant exposure to TYSABRI. For drugs such as interferon and glatiramer acetate, concomitant exposure of this duration was not associated with safety risks in clinical trials. No data are available in MS patients regarding concomitant exposure with immunosuppressant medication. Use of these medicines soon after the discontinuation of TYSABRI may lead to an additive immunosuppressive effect. This should be carefully considered on a case-by-case basis, and a wash-out period of TYSABRI might be appropriate.

\section{Hypersensitivity}

TYSABRI has been associated with hypersensitivity reactions, including anaphylactic/ anaphylactoid reactions, which occurred at an incidence of $<1 \%$. These reactions usually occurred during the infusion or up to 1 hour after completion of the infusion, but there have been occasional post-market reports of delays of up to 2 weeks in symptom onset.

Symptoms associated with these reactions can include urticaria, dizziness, fever, rash, rigors, pruritus, nausea, flushing, hypertension, hypotension, dyspnoea and chest pain. Generally, these reactions are associated with antibodies to TYSABRI.

The risk for hypersensitivity was greatest with early infusions and in patients re-exposed to TYSABRI following an initial short exposure (up to three infusions) and extended period (three months or more) without treatment. However, the risk of hypersensitivity reactions should be considered for every infusion administered.

Patients should be observed during the infusion and for 1 hour after the completion of the infusion. Resources for the management of hypersensitivity reactions should be available.

If a hypersensitivity reaction occurs, discontinue administration of TYSABRI and initiate appropriate therapy. Patients who have experienced a hypersensitivity reaction should not be re-treated with TYSABRI. The possibility of antibodies to TYSABRI should be considered in patients who have hypersensitivity reactions (see ADVERSE EFFECTS, Immunogenicity). 


\section{Immunogenicity}

Disease exacerbations or infusion related events may indicate the development of antibodies against natalizumab (see ADVERSE EFFECTS). If, after approximately 6 months of therapy, persistent antibodies are suspected, they may be detected and confirmed with a subsequent test 6 weeks after the first positive test. Antibodies detected early in the treatment course (e.g. within the first 6 months) may be transient and disappear with continued dosing. Given that efficacy may be reduced or the incidence of hypersensitivity or infusion-related reactions may be increased in a patient with persistent antibodies, physicians should consider the overall benefits and risks of continuing therapy with TYSABRI and cessation of treatment may be appropriate. Patients who receive TYSABRI for a short exposure followed by an extended period without treatment are at higher risk of developing anti-natalizumab antibodies and/or hypersensitivity reactions on re-exposure. Following a prolonged dose interruption (three months or more), consideration should be given to testing for the presence of persistent antibodies (detected on two occasions at least 6 weeks apart) prior to resuming treatment.

\section{Effects on Fertility}

In guinea pigs, intravenous administration of natalizumab was associated with reduced female fertility at an estimated systemic exposure (serum AUC) of 18 times that in humans at the recommended clinical dose, but not at 3 times clinical exposure. Intravenous administration of natalizumab to male guinea pigs did not affect fertility at an estimated exposure 21 times clinical exposure (serum AUC).

\section{Use in Pregnancy (Category C)}

There are no adequate and well-controlled studies of TYSABRI therapy in pregnant women. This drug should be used during pregnancy only if clearly needed. If a woman becomes pregnant while taking TYSABRI, discontinuation of therapy should be considered.

Natalizumab crossed the placenta in guinea pigs and monkeys, but there was no evidence of teratogenicity at respective maternal exposures up to 16 times and 100 times clinical exposure (based on AUC), including effects on early cardiac development (a process known to involve a4 integrins). Intravenous administration of natalizumab to pregnant monkeys during the period of organogenesis was associated with fetal changes (mild anaemia, thrombocytopaenia, increased spleen weights, and reduced liver and thymus weights associated with increased splenic extramedullary haematopoiesis, thymic atrophy and decreased hepatic haematopoiesis), at estimated maternal exposures of 17 times or greater (based on AUC) the clinical exposure at the recommended dose. At the no-effect dose, the extent of maternal exposure was uncertain. Offspring born to monkeys treated intravenously with high doses of natalizumab (100 times clinical exposure based on AUC) showed thrombocytopaenia (reversed upon clearance of natalizumab) and enlarged spleen, but there was no evidence of anaemia.

Intravenous administration of natalizumab to guinea pigs during late gestation and lactation was associated with reduced pup viability, with maternal exposure (based on AUC) estimated at 18 fold clinical exposure. At the no-effect dose, maternal exposure was 3-fold clinical exposure.

\section{Use in Lactation}

TYSABRI has been detected in human milk. Because of this, and because the potential for serious adverse reactions is unknown, a decision should be made whether to discontinue breastfeeding or TYSABRI therapy. 
Intravenous administration of natalizumab to guinea pigs during late gestation and lactation was associated with reduced pup viability, with estimated maternal exposure (AUC) 18-fold that in humans at the recommended clinical dose, and, 3-fold clinical exposure at the no-effect dose.

\section{Use in the Elderly}

Clinical studies of TYSABRI did not include sufficient numbers of patients aged 65 years and over to determine whether they respond differently than younger patients.

\section{Paediatric and Adolescent use}

Safety and effectiveness of TYSABRI in paediatric and adolescent patients with multiple sclerosis below the age of 18 have not been studied. TYSABRI is not indicated for use in paediatric and adolescent patients less than 18 years.

\section{Carcinogenicity}

Natalizumab showed no effects on in vitro assays of $\alpha 4$-integrin positive tumour line proliferation/cytotoxicity. Xenograft transplantation of two $\alpha 4$-integrin positive human tumour lines (leukaemia, melanoma) into immunodeficient mice demonstrated no increase in tumour growth rates or metastasis resulting from natalizumab treatment.

\section{Genotoxicity}

Natalizumab was negative in genotoxicity assays in vitro (mouse lymphoma forward mutation assay, chromosomal aberration in human lymphocytes).

\section{Interactions with Other Medicines}

The safety and efficacy of TYSABRI in combination with antineoplastic or immunosuppressive agents have not been established. Concurrent use of these agents with TYSABRI may increase the risk of infections, including PML and other opportunistic infections (see CONTRAINDICATIONS and ADVERSE EFFECTS, Infections).

In phase 3 clinical trials in multiple sclerosis (Studies 1 and 2), concomitant treatment of relapses with a short course of corticosteroids was associated with an increased rate of infection. However, the increase in infections was similar in TYSABRI-treated patients who received steroids compared with placebo-treated patients who received steroids. Short courses of corticosteroids can be used in combination with TYSABRI.

\section{Immunisations}

In a randomised, open label study of 60 patients with relapsing MS there was no significant difference in the humoral immune response to either a neoantigen (keyhole limpet haemocyanin) or a recall antigen (tetanus toxoid) between patients who were treated with TYSABRI for 6-months compared to an untreated control group. Live vaccines have not been studied. No data are available on the secondary transmission of infection by live vaccines in patients receiving TYSABRI.

\section{Effect on Laboratory Tests}

TYSABRI induces increases in circulating lymphocytes, monocytes, eosinophils, basophils, and nucleated red blood cells. Elevations of neutrophils are not observed. TYSABRI induces mild decreases in haemoglobin levels that are frequently transient. Haematological 
changes persist during TYSABRI exposure but are reversible, returning to baseline levels usually within 16 weeks after the last dose, and are not associated with clinical symptoms.

In addition to those laboratory tests normally required for monitoring patients with multiple sclerosis, blood chemistry, including liver function tests, are recommended for patients with a history of liver disease or active liver disease, during treatment with TYSABRI.

\section{Use in Renal or Hepatic Impairment}

TYSABRI has not been studied in patients with renal or hepatic impairment in clinical trials.

\section{Effects on Ability to Drive or Use Machines}

The effect of TYSABRI on the ability to drive or use machines has not been studied.

\section{ADVERSE EFFECTS}

In placebo-controlled trials in $1617 \mathrm{MS}$ patients treated with TYSABRI for up to 2 years (placebo: 1135), adverse events leading to discontinuation of therapy occurred in $5.8 \%$ of patients treated with TYSABRI (placebo: 4.8\%). Over the 2-year duration of the studies, $43.5 \%$ of patients treated with TYSABRI reported adverse drug reactions (an adverse event judged related to therapy by the investigating physician) (placebo: $39.6 \%$ ).

Table 3 includes adverse events and selected laboratory abnormalities that occurred in Study 1 (Monotherapy Study) at an incidence of at least 1\% higher in TYSABRI-treated patients than was observed in placebo-treated patients.

Table 3. Adverse Events in Study 1 (Monotherapy Study)

\begin{tabular}{|c|c|c|}
\hline $\begin{array}{l}\text { Adverse Events } \\
\text { (Preferred Term) }\end{array}$ & $\begin{array}{c}\text { TYSABRI }^{\circledR} \\
\mathrm{n}=627 \\
\%\end{array}$ & $\begin{array}{c}\text { Placebo } \\
\text { n=312 } \\
\%\end{array}$ \\
\hline \multicolumn{3}{|l|}{ General } \\
\hline Headache & 38 & 33 \\
\hline Fatigue & 27 & 21 \\
\hline Arthralgia & 19 & 14 \\
\hline Chest discomfort & 5 & 3 \\
\hline Acute hypersensitivity reactions ${ }^{\star *}$ & 4 & $<1$ \\
\hline Other hypersensitivity reactions ${ }^{\star *}$ & 5 & 2 \\
\hline Seasonal allergy & 3 & 2 \\
\hline Rigors & 3 & $<1$ \\
\hline Weight increased & 2 & $<1$ \\
\hline Weight decreased & 2 & $<1$ \\
\hline \multicolumn{3}{|l|}{ Infection } \\
\hline Urinary tract infection & 21 & 17 \\
\hline Lower respiratory tract infection & 17 & 16 \\
\hline Gastroenteritis & 11 & 9 \\
\hline Vaginitis* & 10 & 6 \\
\hline Tooth infections & 9 & 7 \\
\hline Herpes & 8 & 7 \\
\hline Tonsillitis & 7 & 5 \\
\hline \multicolumn{3}{|l|}{ Psychiatric } \\
\hline Depression & 19 & 16 \\
\hline
\end{tabular}




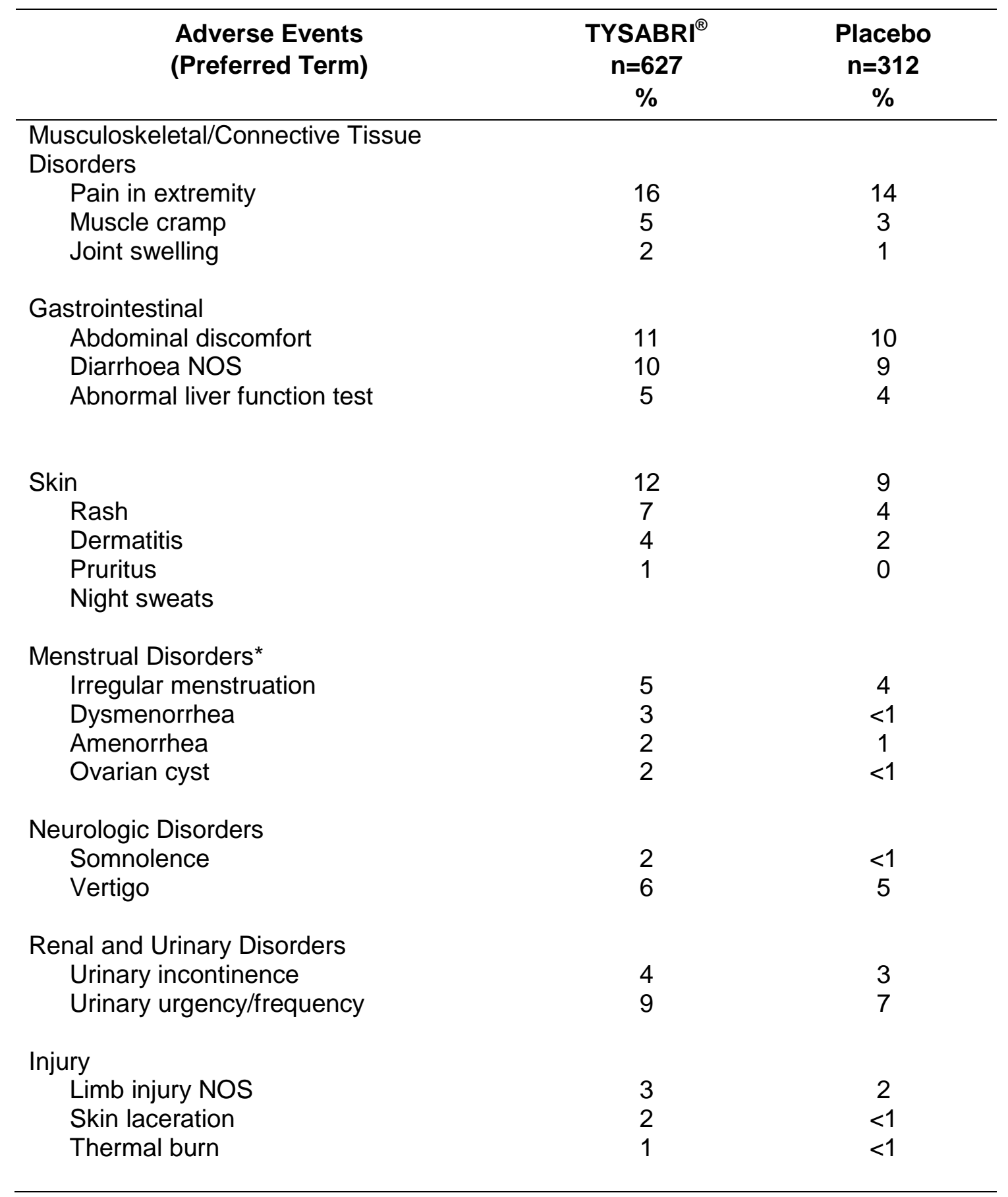

* Percentage based on female patients only

** Acute versus other hypersensitivity reactions are defined as occurring within 2 hours post-infusion versus more than 2 hours

Adverse drug reactions reported with TYSABRI with an incidence of $0.5 \%$ greater than reported with placebo and not already included in Table 3 are shown below. The reactions are reported as MedDRA preferred terms under the MedDRA primary system organ class. Frequencies were defined as follows:

Common (>1/100, $\leq 1 / 10)$, uncommon $(>1 / 1000, \leq 1 / 100)$.

Infections and infestations
Common
Nasopharyngitis

Immune system disorders

Common

Urticaria 
Nervous system disorders Common

\section{Dizziness}

Gastrointestinal disorders

$\begin{array}{ll}\text { Common } & \text { Vomiting } \\ & \text { Nausea }\end{array}$

General disorders and administration site conditions

Common

Pyrexia

No trials were conducted directly comparing the adverse event profile of TYSABRI plus AVONEX to TYSABRI alone. When comparing across trials, in general, adverse events appeared to be more common in those receiving TYSABRI in combination with AVONEX than those receiving TYSABRI alone (99.2\% vs. 95.1\%, TYSABRI plus AVONEX vs TYSABRI, respectively). Many of these differences appeared to be attributable to adverse events often associated with beta-interferon (headache, fatigue, depression, arthralgia, flulike symptoms). Peripheral oedema and herpes viral infections were slightly more common in those receiving TYSABRI in combination with AVONEX than those receiving TYSABRI alone. The overall incidence of serious adverse events and serious infections were similar in those receiving TYSABRI in addition to AVONEX as compared with TYSABRI alone, although appendicitis was slightly more common in those receiving combination treatment $(0.8 \%$ vs. $0.2 \%)$.

\section{Infusion-Related Reactions}

In 2-year controlled clinical trials in MS patients (Studies 1 and 2), an infusion-related event was defined as an adverse event occurring during the infusion or within 1 hour of the completion of the infusion. Approximately $24 \%$ of TYSABRI-treated MS patients experienced an infusion-related reaction, compared to $18 \%$ of placebo-treated patients. Infusion-related reactions in TYSABRI-treated patients included headache, dizziness, fatigue, rigors and localised or multi-symptomatic hypersensitivity reactions.

\section{Hypersensitivity}

The majority of hypersensitivity reactions are infusion-related. No delayed hypersensitivity reactions were seen in 2-year controlled clinical studies in MS patients (Studies 1 and 2). In Studies 1 and 2, hypersensitivity reactions occurred in up to $4 \%$ of patients. This includes acute urticaria, which was observed in approximately $2 \%$ of patients.

Anaphylactic/anaphylactoid reactions occurred in $<1 \%$ of patients. All patients recovered with treatment and/or discontinuation of the infusion.

Patients who became persistently positive for antibodies to TYSABRI were more likely to have an infusion-related reaction than those who were antibody-negative (see ADVERSE EFFECTS, Immunogenicity).

\section{Immunogenicity}

Patients in Study 1 and Study 2 were tested for antibodies to natalizumab every 12 weeks. Antibodies against natalizumab were detected in approximately $10 \%$ of multiple sclerosis patients receiving TYSABRI in 2-year controlled clinical trials in MS patients. Persistent anti-natalizumab antibodies (one positive test reproducible on retesting at least 6 weeks later) developed in approximately $6 \%$ of patients. Antibodies were detected on only one occasion in an additional $4 \%$ of patients. Approximately $90 \%$ of patients who became persistently antibody-positive by this assay had developed detectable antibodies by 12 weeks. Anti-natalizumab antibodies were neutralizing in vitro. 
Persistent antibodies to natalizumab were associated with a substantial decrease in the effectiveness of TYSABRI and an increased incidence of hypersensitivity reactions. The risk of disability progression and the annualised relapse rate of persistently antibody-positive TYSABRI-treated patients were similar to the rates in subjects who received placebo.

Infusion-related reactions most often associated with persistent antibody-positivity included urticaria, rigors, nausea, vomiting, and flushing. Additional adverse events more common in persistently antibody-positive patients included myalgia, hypertension, dyspnoea, anxiety, and tachycardia.

If, after approximately 6 months of therapy, persistent antibodies are suspected, they may be detected and confirmed with a subsequent test 6 weeks after the first positive test. Given that efficacy may be reduced or the incidence of hypersensitivity or infusion-related reactions may be increased in a patient with persistent antibodies, physicians should consider the overall benefits and risks of therapy with TYSABRI and cessation of treatment may be appropriate.

\section{Infections}

In 2-year controlled clinical trials in MS patients, the rate of infection was approximately 1.5 per patient-year in both TYSABRI-treated patients and placebo-treated patients. The nature of the infections was generally similar in TYSABRI-treated and placebo-treated patients. The infections were predominately upper respiratory tract infections, influenza and urinary tract infections. Most patients did not interrupt treatment with TYSABRI during the infection and recovery occurred with appropriate treatment.

Cases of progressive multifocal leucoencephalopathy (PML) have been reported in clinical trials. In Study 2 (Add-On Study), two cases including one fatality, occurred in MS patients who were being treated with concomitant interferon beta-1a therapy for more than 2 years. In another trial, one patient with Crohn's disease and chronic lymphopenia due to azathioprine developed PML and died. This patient had stopped concomitant immunosuppressive agents, including azathioprine, at least 6 months prior to the development of neurological symptoms. There is a risk of PML associated with TYSABRI alone. In the post-marketing setting, additional cases of PML in patients treated with TYSABRI monotherapy have been reported (see BOXED WARNING and PRECAUTIONS, Progressive Multifocal Leucoencephalopathy).

PML has been reported following discontinuation of TYSABRI in patients who did not have findings suggestive of PML at the time of discontinuation. Patients and healthcare professionals should continue to be vigilant for any new signs or symptoms that may be suggestive of PML for approximately six months following discontinuation of TYSABRI.

The only opportunistic infection in multiple sclerosis clinical trials was a case of cryptosporidial gastroenteritis with a prolonged course. In clinical studies for other indications, opportunistic infections (e.g. pneumocystis carinii pneumonia, pulmonary mycobacterium avium intracellulare, bronchopulmonary aspergillosis) were observed uncommonly in TYSABRI-treated patients; the majority of these patients either were receiving concurrent immunosuppressants or had major co-morbidities.

In clinical trials, herpes infections occurred slightly more frequently in patients treated with TYSABRI than in patients treated with placebo. In post-marketing experience, serious lifethreatening, and sometimes fatal cases of encephalitis and meningitis caused by herpes simplex or varicella zoster have been reported in multiple sclerosis patients receiving TYSABRI. The duration of treatment with TYSABRI prior to onset ranged from a few months to several years. If herpes encephalitis or meningitis occurs, TYSABRI should be discontinued and appropriate treatment for herpes encephalitis or meningitis should be administered. 


\section{Malignancies}

No differences in incidence rates or the nature of malignancies between TYSABRI and placebo-treated patients were observed over 2 years of treatment. However, observation over longer treatment periods is required before any effect of TYSABRI on malignancies can be excluded.

\section{Post-Marketing Experience}

Clinically significant liver injury has been reported in patients treated with TYSABRI in the postmarketing setting (see PRECAUTIONS - Hepatotoxicity).

PML has been reported in patients treated with TYSABRI monotherapy in the post-marketing setting. An estimate of the different levels of risk for PML in different patient subgroups is shown in table 4 below.

Table 4: Post-marketing data on PML risk in different patient subgroups

\begin{tabular}{|l|l|l|l|l|}
\hline \multirow{2}{*}{$\begin{array}{c}\text { Anti-JCV } \\
\text { Antibody } \\
\text { Negative }\end{array}$} & \multirow{2}{*}{$\begin{array}{c}\text { Tysabri } \\
\text { Exposure }\end{array}$} & \multicolumn{2}{|c|}{ Anti-JCV Antibody Positive } \\
\cline { 3 - 5 } & & $\begin{array}{l}\text { No Prior } \\
\text { Immunosuppressant Use }\end{array}$ & $\begin{array}{l}\text { Prior } \\
\text { Immunosuppressant } \\
\text { Use }\end{array}$ \\
\hline $0.1 / 1,000$ & & $1 / 1,000$ & $2 / 1,000$ \\
\cline { 3 - 5 } & $1-24$ infusions & $5 / 1,000$ & $11 / 1,000$ \\
\cline { 3 - 5 } & $25-48$ infusions & $6 / 1,000$ & $9 / 1,000$ \\
\cline { 3 - 5 } & $49-72$ infusions & &
\end{tabular}

The PML risk estimates are based on post-marketing data from approximately 125,000 TYSABRI-exposed patients

Data beyond 6 years of treatment are limited

In post-marketing experience, there have been reports of eosinophilia (eosinophil count $>$ $1,500 / \mathrm{mm}^{3}$ ) without clinical findings. In cases where TYSABRI therapy was discontinued the elevated eosinophil levels resolved.

Serious, rare cases of haemolytic anaemia have been reported in patients treated with TYSBARI in post marketing observational studies.

\section{DOSAGE AND ADMINISTRATION}

TYSABRI therapy is to be initiated and supervised by neurologists, in centres with timely access to MRI.

\section{Adults}

The recommended dose of TYSABRI is $300 \mathrm{mg}$ administered by IV infusion every four weeks. Dilute TYSABRI concentrate $300 \mathrm{mg} / 15 \mathrm{~mL}$ in $100 \mathrm{~mL} 0.9 \%$ Sodium Chloride Injection, and infuse over approximately one hour. Do not administer TYSABRI as an IV push or bolus injection (see Preparation Instructions).

Observe patients during the infusion and for 1 hour after the infusion is complete. Promptly discontinue the infusion upon the first observation of any signs or symptoms consistent with a hypersensitivity-type reaction (see PRECAUTIONS, Hypersensitivity). Staff and facilities should be available for treating anaphylaxis, in the unlikely event that this occurs. 


\section{Children and adolescents ( $<18$ years)}

Safety and effectiveness of natalizumab in MS patients below the age of 18 years of age have not been studied. TYSABRI is not indicated for children and adolescents.

\section{Renal and hepatic impairment}

No formal pharmacokinetic studies have been conducted to examine the effects of renal or hepatic impairment. The mechanism for elimination and results from population pharmacokinetics suggest that dose adjustment would not be necessary in patients with renal or hepatic impairment.

\section{Preparation Instructions}

TYSABRI (natalizumab) is free of preservatives. Use aseptic technique when preparing TYSABRI solution for IV infusion. TYSABRI is for single use in one patient only. Discard any residue.

TYSABRI is a colourless, clear to slightly opalescent concentrate. Inspect the vial for particulate material prior to dilution and administration. If visible particulates are observed and/or the liquid in the vial is discoloured, the vial must not be used. Do not use TYSABRI beyond the expiration date on the carton or vial.

To prepare the solution, withdraw $15 \mathrm{~mL}$ of TYSABRI concentrate from the vial using a sterile needle and syringe. Inject the concentrate into $100 \mathrm{~mL} 0.9 \%$ Sodium Chloride Injection. No other IV diluents may be used to prepare the TYSABRI solution.

Gently invert the TYSABRI solution to mix completely. Do not shake. Inspect for particulate material prior to administration.

Following dilution, infuse TYSABRI solution immediately or within 8 hours if stored at $2^{\circ} \mathrm{C}$ to $8^{\circ} \mathrm{C}$ and protected from light. If stored at $2^{\circ} \mathrm{C}$ to $8^{\circ} \mathrm{C}$, allow the solution to warm to room temperature prior to infusion. Do not freeze.

\section{Administration Instructions}

Infuse TYSABRI $300 \mathrm{mg}$ in $100 \mathrm{~mL}$ 0.9\% Sodium Chloride Injection over approximately one hour. After the infusion is complete, flush with $0.9 \%$ Sodium Chloride Injection.

Use of filtration devices during administration has not been evaluated. Other medications should not be injected into infusion set side ports or mixed with TYSABRI.

\section{OVERDOSAGE}

Safety of doses higher than $300 \mathrm{mg}$ has not been adequately evaluated. The maximum amount of TYSABRI that can be safely administered has not been determined.

Contact the Poisons Information Centre on 131126 for advice on management of overdosage.

\section{PRESENTATION AND STORAGE CONDITIONS}


TYSABRI concentrated injection solution contains $300 \mathrm{mg} / 15 \mathrm{~mL}$ natalizumab in a sterile, single-use vial free of preservatives (packs of 1 vial). TYSABRI is for single use in one patient only.

TYSABRI single-use vials must be stored between $2^{\circ} \mathrm{C}$ to $8^{\circ} \mathrm{C}$. Do not use after the expiration date on the carton and vial label. DO NOT SHAKE OR FREEZE. Protect from light (store in carton).

If not used immediately, diluted solution can be stored at $2^{\circ} \mathrm{C}$ to $8^{\circ} \mathrm{C}$, protected from light. TYSABRI solution for infusion must be administered within 8 hours of preparation.

\section{SPONSOR}

Biogen Australia Pty Ltd

ABN 30095760115

Level 3, 123 Epping Road

North Ryde NSW 2113

Date of First Inclusion in the ARTG: 1 November 2006

Date of most recent amendment: 11 Jan 2016

AVONEX $^{\circledR}$, TYSABRI $^{\circledR}$ and BIOGEN ${ }^{\circledR}$ are registered trademarks of Biogen MA Inc. 